Review

\title{
Under pressure: Cerebrospinal fluid contribution to the physiological homeostasis of the eye
}

\author{
Serena Mirra ${ }^{\mathrm{a}, \mathrm{b}}$, Gemma Marfany ${ }^{\mathrm{a}, \mathrm{b}}$, Jordi Garcia-Fernàndez ${ }^{\mathrm{a}, *}$ \\ ${ }^{a}$ Department of Genetics, Microbiology and Statistics, Faculty of Biology, and Institut de Biomedicina (IBUB), University of Barcelona, Barcelona, Spain \\ ${ }^{\mathrm{b}}$ Centro de Investigación Biomédica en Red de Enfermedades Raras (CIBERER), Spain
}

\section{A R T I C L E I N F O}

\section{Keywords:}

CSF pressure

Lamina cribrosa

Optic nerve

Glymphatic system

Ciliopathies

Glaucoma

\begin{abstract}
A B S T R A C T
The cerebrospinal fluid (CSF) is a waterly, colorless fluid contained within the brain ventricles and the cranial and spinal subarachnoid spaces. CSF physiological functions range from hydromechanical protection of the central nervous system (CNS) to CNS modulation of developmental processes and regulation of interstitial fluid homeostasis. Optic nerve (ON) is surrounded by CSF circulating in the subarachnoid spaces and is exposed to both CSF (CSFP) and intra ocular (IOP) pressures, which converge at the lamina cribrosa (LC) as two opposite forces. The trans-lamina cribrosa pressure gradient (TLPG) is defined as IOP - CSFP and its alterations (due either to an elevation in IOP or a reduction in ICP) could result in structural damaging of the ON, including glaucomatous changes.

The purpose of this review is to update the readers on the CSF contribution in controlling the functions/ dysfunctions of $\mathrm{ON}$ by regulating homeostasis at LC. We also highlight emerging parallelisms regarding the expression of cilia-related genes in the regulation of common functions of body fluids in both brain and eye structures.
\end{abstract}

\section{Introduction}

CSF is the nutrient-rich fluid that bathes the brain and spinal cord. It is continuously secreted into the cerebral ventricles by the choroid plexus (CP), a layer of surrounding a core of capillaries, and it is absorbed into the venous system. CSF plays an essential role in the homeostasis of the interstitial fluid of the brain parenchyma and the regulation of neuronal function [1-3]. Indeed, CSF composition is tightly controlled and includes amino acids, vitamins, minerals, ions, growth factors and signalling molecules that are kept at very specific concentrations depending on the developmental stage [4]. Additionally, CSF helps to cushion the central nervous system from mechanical injury. Alterations in CSF dynamics and composition are responsible for the major alterations of cerebral homeostasis and physiology observed in hydrocephalus and dementia disorders [5].

A first mention of a mysterious fluid inside the brain is found in the writings of the ancient Egyptians (1700 BCE) [6]. In Greece,
Hippocrates (129-219 AD) also reported the presence of "water inside the head" in hydrocephalus subjects, while Galen of Pergamon (129-200/216 CE) described a "vaporous and smoky" secretion at the base of the brain, arising from the blood vessels as a "vital spirit". The succession of events orchestrating the development of brain ventricular cavity-CSF system is quite uniform across mammalian species. The CSF formation rate appears to increase gradually during ontogenesis, supporting the functional importance of the CSF in regulating the development of CNS [7]. The CSF formation rate has been measured, for example, in turtles $(1.4 \mu \mathrm{l} / \mathrm{min})$, rats $(2.2 \mu \mathrm{l} / \mathrm{min})$, rabbits $(8-11 \mu \mathrm{l} /$ $\mathrm{min})$, cats $(14-21 \mu \mathrm{l} / \mathrm{min})$, monkeys (19-35 $\mu \mathrm{l} / \mathrm{min})$, and goats $(160 \mu \mathrm{l} /$ $\min \mu \mathrm{l} / \mathrm{min}$ ) $[8,9]$. In rats and many other species the gradual increase of CSF formation during postnatal development correlates with postnatal morphological and functional maturation of choroidal epithelium [10]. However, the ontogeny and phylogeny of the "vital spirit" around which the vertebrate brain is organised is not fully understood yet. Beyond vertebrates, the CSF system is hypothesized to appear in the

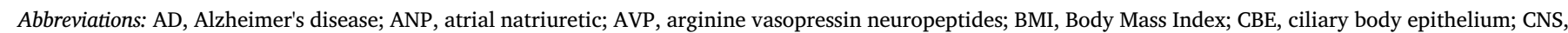

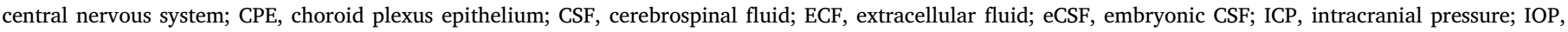

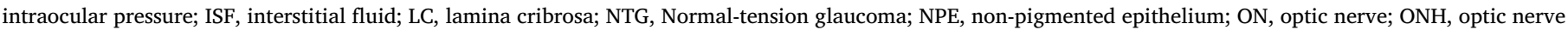

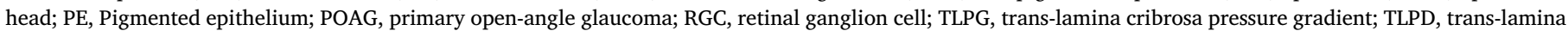
cribrosa pressure difference

* Corresponding authors.

E-mail addresses: serena.mirra@ub.edu (S. Mirra), gmarfany@ub.edu (G. Marfany), jordigarcia@ub.edu (J. Garcia-Fernàndez). 


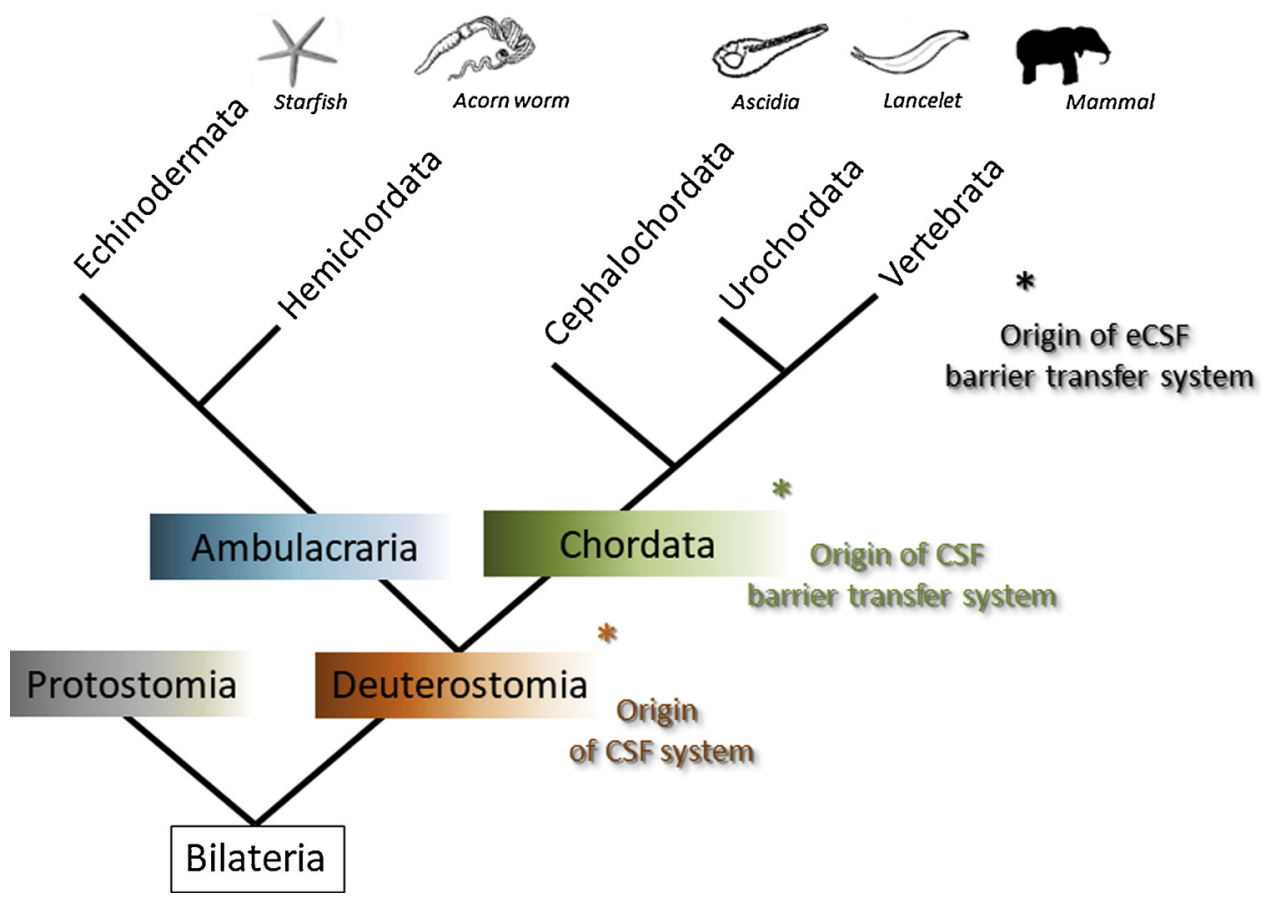

Fig. 1. Phylogeny and evolution of CSF. Metazoan phylogeny classifies animals with bilateral symmetry in Protostomia and Deuterostomia. Protostomia includes clades Priapulida, Nematoda, Arthopoda, Platyhelminthes, Mollusca and Annelida. The sister group of Protostomia are the Deuterostomia, including Ambulacraria (Echinodermata and Hemichordata) and Chordata (Urochordata, Cephalochordata and Vertebrata). Evolution of CSF system across the different phyla and subphyla is indicated (*).CSF, cerebro-spinal fluid; eCSF, embryonic cerebro-spinal fluid.

deuterostome lineage as a way to maintain the chemical environment required for the functioning of CNS cells, including the neuroendocrine pathways. Earlier in the phylum Chordata, the cephalochordate amphioxus includes a larval stage in which the CNS is open through the neuropore, thus allowing seawater to enter the ventricular lumen. However, after metamorphosis, the anterior neuropore closes and the brain becomes a closed system, with a proper CSF regulating brain development and homeostasis [11]. Strikingly, one main innovation at early stages of vertebrate origins was probably a heterochronic switch to an early closure of the neuropore during embryogenesis (and also, the secretion of an embryonic-eCSF distinct from the adult CSF), which translated into an early isolation of the brain cavities from the surrounding external media, hence enabling a higher level of internal control and regulation (Fig. 1; reviewed in [12] and [13]).

The ON develops from the optic vesicle, an outpocketing of the forebrain, and can be considered part of the CNS [14]. As a CNS structure, the ON is myelinated by oligodendrocytes, ensheathed in the cranial meningeal layers and surrounded by CSF circulating within the subarachnoid space [14]. Recently, many studies have demonstrated that an imbalanced pressure exercised by CSFP induce structural damage of the ON $[15,16]$. CSFP, together with IOP, create a pressure gradient at the LC, a specialized region at the optic nerve head (ONH). TLPG imbalance could be a major determinant for the pathophysiology of several eye diseases, such as glaucoma.

In this review, we aim to describe what is known about CSF physiological contribution to maintain LC homeostasis and its potential as a pathophysiologic factor in ONH diseases. We highlight the CSF theory of glaucoma, a collection of diseases characterized by progressive degeneration of the ON cells that represent a leading cause of irreversible blindness worldwide. Finally, we highlight emerging parallelisms in the expression of genes controlling the function/dysfunction of body fluids circulating in both brain and neurosensory organs.

\section{Cerebrospinal fluid: an extraordinarily dynamic fluid flowing through CNS}

The developmental processes giving rise to the highly compartmentalized CNS architecture are complex and require a series of exquisitely-regulated events. The vertebrate ventricular system develops from the single cavity inside the neural tube. During the process of neurulation, the neural plate folds longitudinally and the amniotic fluid is captured inside the nascent neural tube. The center of the neural tube forms the ventricular space, surrounded by a pseudo-monostratified neuroepithelial cells that rapidly divide to expand the neural progenitor cell pool [17]. The first CP appears shortly after the neural tube closure in the fourth ventricle as a specialized vascular epithelium producing CSF. CSF regulates cell replication, cell survival and neuronal differentiation, through both pressure and biochemical mechanisms $[11,18,19]$. The subsequent neuroepithelium patterning and regionalization will give rise to the distinct regions of the mammalian brain. After the formation of the ventricular system, CSF flows from the $\mathrm{CP}$ through the lateral ventricle, the interventricular foramen of Monro, the third ventricle, the cerebral aqueduct of Sylvius and the fourth ventricle. Therefore, CSF fills the central canal of the spinal cord and is passively absorbed into the venous system. Importantly, embryonic CSF (eCSF) contains three-fold more protein than the adult CSF, further highlighting its important roles during embryonic development [20].

Homeostasis of the neural microenvironment depends on the effective separation of this environment from the blood. In parallel, a sophisticated regulation of the electrolyte balance, an efficient exchange of metabolic waste products and a dynamic circulation of nutrients, are also required. The CSF meets these requirements and acts as a key interface that guarantees both hydromechanical protection and neuraxis compartmentalisation, even so allowing dynamic exchanges.

\subsection{Cerebrospinal fluid production and composition}

In the human brain, about $75 \%$ of CSF is secreted by the CP, and the additional $25 \%$ is contributed by the extracellular fluid (ECF) of the neuropil, with which the CSF extensively communicates through the ependymal cells layer.

Normal CSF production is $0.20-0.35 \mathrm{ml} / \mathrm{min}$ or $600-700 \mathrm{ml}$ per day. Total volume of CSF in an adult is about $150 \mathrm{ml}$, distributed between $125 \mathrm{ml}$ in cranial and spinal subarachnoid spaces and $25 \mathrm{ml}$ in the brain ventricles. Pulsations coming from arterial CP and ependymal cells cilia allow CSF circulation. On the other hand, CSF can be absorbed into the venous circulation as well as into lymphatic vessels [21]. 
CSF is composed of $99.13 \%$ of water, with a $\mathrm{pH}$ of 7.33 . Other components are proteins $(35 \mathrm{mg} / \mathrm{dL})$, glucose $(60 \mathrm{mg} / \mathrm{dL})$, Sodium (114.0 mEq/L), Potassium (3.00 mEq/L), Magnesium (2.0-2.5 mEq/L), Chloride (119 mEq/L). Several studies have demonstrated the existence of chronobiological variations in CSF secretion, probably related to circadian differences in the activation of the autonomous nervous system [22,23]. CSF from the adult lumbar region contains $15-45 \mathrm{mg}$ / $\mathrm{dl}$ protein and CSF cell count ranges from 0 to 5 cells per milliliter. Biochemical and physical examination of the CSF chemical contents may provide important diagnostic information in a number of medical conditions. For instance, the 42 aa form of beta amyloid (Aß42) and both total- and phosphorylated tau (t-tau and p-tau) concentration in CSF can be used as Alzheimer's disease (AD) biomarkers [24,25].

\subsection{Cerebrospinal fluid pressure}

CSFP is defined as the intracranial pressure in the prone position. The normal range of CSFP is defined between 10 and $15 \mathrm{mmHg}$ in adults and 4.4 and $7.3 \mathrm{mmHg}$ in newborns. CSFP variations are most due to jugular venous pressure, pulse wave, respiratory cycle, physical activity and posture. The first procedures described to drain CSF by lumbar puncture date back to the end of the nineteenth century [26]. Currently, CSF pressure can be measured by invasive techniques involving pressure transducers placed in the brain parenchyma or connected to CSF spaces via an external drain. The development of noninvasive methods is limited to using a transcranial Doppler ultrasound system or recording the electrophysiological activity of tympanic membranes [27]. More recent studies described a reliable non-invasive method to predict CSFP based on trans-ocular venous and arterial hemodynamic measurements [28]. Other groups have described multivariate algorithms-based methods incorporating systemic parameters such as age, blood pressure and body mass index as indirect indicators of CSFP [29]. All the physiological processes regulating CSF secretion, circulation and absorption are involved in CSFP determination. For instance, the CSF concentration of both atrial natriuretic and arginine vasopressin neuropeptides (ANP and AVP) are known to increase with increasing CSFP, as it does the expression of these hormone receptors in the choroidal epithelium. The final effect is a reduction of CSF choroidal secretion, which in turn induces a dilatation of pial arteries, with a consequent reduction of cerebral perfusion pressure [30]. However, we are still far from a comprehensive understanding of the mechanisms regulating CSFP.

\section{The relevance of cerebrospinal fluid for eye physiology}

Retina, the light sensing tissue lining the inner surface at the back of the eye, matures from an evagination of the brain during fetal development and can be considered an integral part of the CNS. Light stimuli processed by photoreceptor cells in the retina are transmitted throughout the retinal neuronal network until reaching the retinal ganglion cells (RGC), whose axons form the ON, a cable-like grouping of nerve fibers. The ON leaves the intracranial space, extends from the optic disc to the optic chiasma and transmits the vision signals to the lateral geniculate body, where visual information is relayed to the brain visual cortex.

The orbital portion of the ON is surrounded by CSF circulating within the intracranial subarachnoid space. LC is the structural element of the $\mathrm{ONH}$ and it represents a barrier between the intraocular space, which has a higher pressure, and retrobulbar space, with a lower pressure. Indeed, a TLPG is formed across the LC [31-33] (Fig. 2).

The theory of glaucoma being a two-pressure disease was initially proposed by Volkov in the 1970s, and more recently by Berdahl and Ren [15,34-36]. They wondered whether abnormal pressure outside the eye might cause normal tension glaucoma, a condition in which the glaucomatous subjects have normal intraocular pressure (IOP), while CSFP is lower than normal [37]. This theory was supported by studies

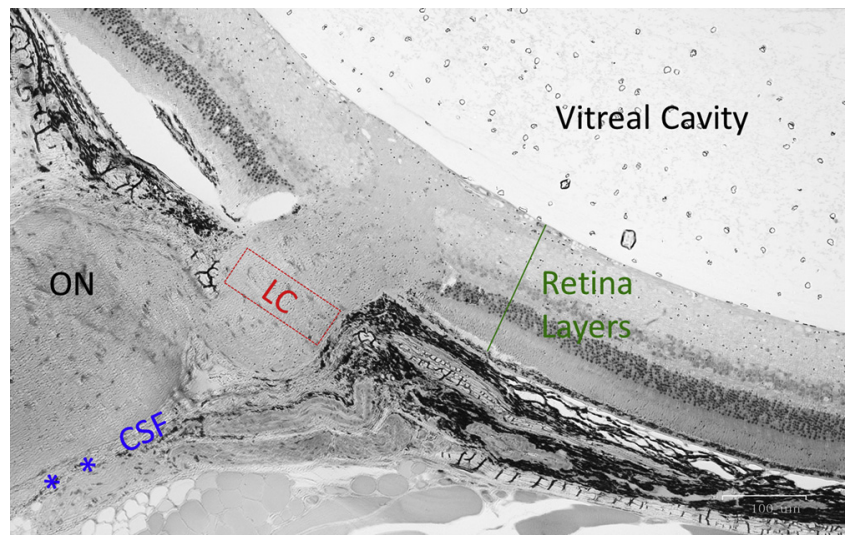

Fig. 2. Histologic anatomy of a normal optic head nerve. The LC (red) is a thin structure located at the base of the ON. The nerve fibres exit the retinal neuronal layers (green) and run through the lamina cribrosa pores. The ON is surrounded by CSF (blue) flowing through the intracranial subarachnoid space.

conducted in astronauts, which commonly experience visual disturbances with disc swelling. Six month-long space flights frequently lead the astronauts to develop a clinical syndrome named spaceflightassociated neuro-ocular syndrome (SANS), bearing marked similarities to idiopathic intracranial hypertension with papilloedema [38]. This phenotype could be explained as a consequence of the absence of gravity, which allows CSF to diffuse equally through the intracranial subarachnoid space, thus increasing venous pressures and producing a higher CSFP at the eye [39].

In summary, ONH healthy state depends on the balance between IOP, CSFP and the mean arterial blood pressure. Therefore, a strong interest is emerging concerning the influence of CSFP upon the pressure gradient at ONH. Changes in this gradient may be a fundamental component in disorders such as glaucoma, idiopathic intracranial hypertension and venous occlusions.

\subsection{LC anatomy: pressure gradient forces at stake}

LC is a thin porous tissue formed by collagen-rich "beams" surrounding capillaries. It is located at the base of $\mathrm{ON}$, where the fibres exit the eye through a hole in the sclera [40]. The LC has an average diameter of $1.7 \mathrm{~mm}$ and is formed by $200-400$ pores of varying sizes through which approximately 1 million axons exit the eye [41]. LC is principally composed by laminin, elastin and collagens, and provides mechanical support [42]. Moreover, LC is the substrate for two types of cells that provide homeostatic and trophic support to the axons [43]. The first population, formed by GFAP-positive cells with multiple long and thin cellular processes, are identified as astrocytes. They draw nutrients from capillaries that run within the connective tissue beams. A second cell type, characterized by a large, flat morphology and negative for GFAP, are named LC cells. They share behavioural and molecular features with astrocytes population, which suggest that LC cells are also a subtype of astrocytes [44].

The posterior part of the LC faces the anterior region of the ONH. Importantly, this ON region is unmyelinated and closely associates with astrocytes. These features entail specific metabolic requirements and differential responses to metabolic stress, as we will describe below.

As part of the CNS, the ON is surrounded by the meninges and CSF, which also exercises a force upon the $\mathrm{ONH}$. The internal pressure within the globe of the eye (IOP) and external pressure exerted by the tissues surrounding the posterior region of the eye converge on the LC, creating a region of stress concentration surrounding the ONH [45]. The translamina cribrosa pressure difference (TLPD) is defined as the difference between the IOP and the retrobulbar pressure [33], and this parameter is relevant for the physiology and pathophysiology of the ONH. TLPD 
a

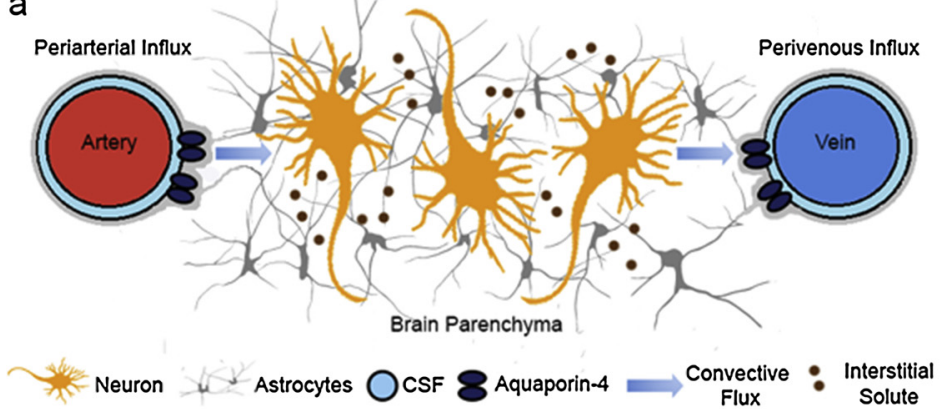

b

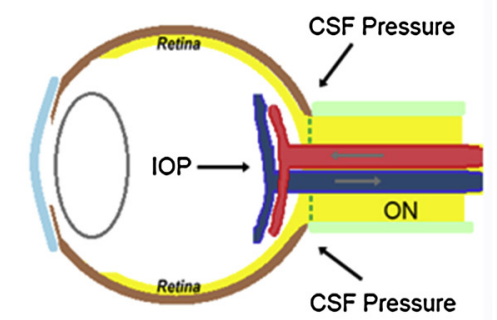

Para-arterial CSF Flux Subrachnoid Space Paravenous clearance Flux Lamina Cibrosa

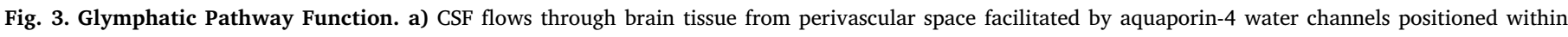

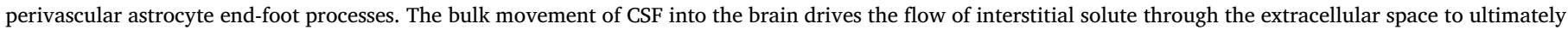
collect within perivenous spaces. b) Schematic representation of the "ocular glymphatic system" hypothesis postulated by Wostyn et al. [70].

alterations are presumed to cause axonal damage in glaucoma, leading to either structural damage on the retinal ganglion cells axons (mechanical hypothesis), with consequent neuroinflammation and alterations in axonal transport dynamics, and/or constriction of blood flow within the lamina's tissue (hemodynamical hypothesis) [46-49].

Remarkably, LC is a dynamic component in vivo. The LC biomechanical properties allow movements in both sagittal and coronal directions. Sagittal movements are linked to pulse-synchronous changes in the TLPD and are mechanically limited by the ONH. On the other hand, coronal movement are induced by central retinal vein pulsations. Importantly, CSFP contributes to calibrate the LC movement, even if indirectly, since it influences both TLPD and the central retinal vein pressure. Regarding the physiological function of LC movements, several hypotheses have been postulated, e.g. some groups hypothesize that LC movements would follow through and facilitate orthograde and retrograde axoplasmic flow through the eye [50-52]. Therefore, even if the physiological importance of LC positional oscillations is still far to be elucidated, CSFP may play a pathophysiological role in this context.

\subsection{Metabolic stress at $L C$}

Axonal changes described in glaucoma animal models satisfactorily resume the complex spectrum of physiological alterations occurring at ONH when the TLPG is impaired. One of the first alterations observed in early stages of glaucoma is the inhibition of axonal transport at LC, with consequent accumulation of organelles. Organelle arrest has been described in both $\mathrm{ONH}$ prelaminar and postlaminar regions in monkey models of experimental glaucoma [53], as well as in the glial lamina of rat models of early-onset glaucoma [54]. This phenomenon can be correlated with molecular changes involving motor proteins and cytoskeleton structures that are vital for axonal organelle trafficking. Significant changes in cytoskeletal protein levels have been described in pig and rat models of glaucoma [55]. Kinesin-1 levels decrease significantly in the prelaminar ONH during ocular hypertension [56], whereas dynein levels become elevated in the retrolaminar region [57]. The mechanism hypothesized to explain the blockages of axonal transport at the LC is the hiperactivation of kinesin-1 degradation pathway, leading to the lamina obstruction and/or cell soma disconnection.

RGC survival and function are highly dependent on the energy supplied by mitochondrial activity. The absence of myelinisation makes the axons at LC highly energy demanding. This condition is reflected by the high mitochondrial concentration at both prelaminar and laminar regions of RGC axons [58]. Elevated IOP may locally compromise the blood supply or its regulation in the lamina, contributing to metabolic stress. Cytochrome oxidase activity increases in the collagenous lamina region of the pig ON when TLPG increases, indicating a significant growth of metabolic requirements in this region [32]. High IOP directly impacts mitochondrial functions in $\mathrm{DBA} / 2 \mathrm{~J}$ mice by promoting reduction of cytochrome c oxidase IV subunit 1, and leading to a decrease in ATP levels and cristae depletion. In addition, alterations in proteins regulating mitochondrial dynamics, such as OPA1 and Dnm1, have been described in animal models with increased IOP [59-61].

\subsection{The ocular glymphatic system: a recent, intriguing hypothesis}

The glymphatic system is an exquisitely organized brain-wide mechanism by which CSF flows through a para-arterial route to enter the brain parenchyma. This pathway allows the potentially toxic metabolic products to be removed from the interstitial fluid (ISF) in the brain and be returned to the CSF and, eventually, to the lymphatic system. It is largely known that CNS blood vessels are covered by astrocytes and surrounded by perivascular space, and several hypotheses about perivascular space significance have been proposed over many years, until 2012, with the work by Iliff et al. These authors used in vivo two-photon and ex vivo fluorescence imaging to demonstrate that tracers injected in CSF could be transported along periarterial space [62]. Then, the CSF influx flows into brain parenchyma driven by arterial pulsation forces where it merges with ISF [63]. The mixed CSF and ISF with interstitial metabolic waste flow towards paravenous pathways and reach the lymphatic system (Fig. 3.a). Further data supporting the possible implication of glymphatic system dysfunctions in promoting human disease were later provided. They also observed that amyloid-beta peptide and Tau proteins could be partially eliminated from the brain parenchyma through the perivenous spaces [62,64]. Aquaporin-4 (AQP4) water channels, abundantly expressed in astrocytic end-foot processes ensheathing the cerebral vasculature, guarantees glymphatic clearance. In mice lacking the AQP4 gene, amyloid-beta clearance is reduced by approximately 55\% [64].

An additional function carried out by the glymphatic system may concern the correct distribution of non-waste compounds, such as glucose, lipids, amino acids, and neurotransmitters in the brain [65].

A rapidly evolving literature is proposing that an "ocular glymphatic system" also exists in the eye and may be relevant to a large number of ocular diseases [66]. Yet the eye, like the brain, has no traditional lymphatic vessels while still needing to clear potentially neurotoxic protein waste. Furthermore, the presence of AQP4 in human ON sections has been recently demonstrated [67]. Several animal models were used over the years to inject tracers into CSF to be detected in the ON [68]. However, the route of entry was neither described nor assumed to be free diffusion from the subarachnoid space. The first evidence of CSF entry through the ON along paravascular spaces was obtained in mice [69]. CSF was postulated to flow through a para-arterial CSF route around the central retinal artery and enter into the paravascular spaces of the retina; then, a para-venous clearance efflux route might travel around the central retinal vein [70] (Fig. 3.b). In this scenario, the LC may mechanically interfere with the glymphatic flow between the ON and retina, at least in glaucomatous eyes, where the LC 
structure is highly altered. As a consequence, the elimination of neurotoxic substances from the retina would be impaired, ulteriorly promoting the glaucomatous damage. Consistently, studies in glaucomatous animal models showed that $\mathrm{A} \beta$ is a likely mediator of pressureinduced RGC death [71]. Therefore, the discovery of a glymphatic system in the eye may be particularly relevant to understand the mechanisms underlying several neurodegenerative ocular diseases. Yet, the existence of an ocular glymphatic system in the human eye remains only a hypothesis, and much more studies are needed to validate it.

\section{CSF contribution to glaucomatous ON damage}

Normal-tension glaucoma (NTG) is a condition characterised by visual field loss and optic disc excavation identical to primary openangle glaucoma (POAG) despite apparently normal IOP. Initially, NTG was related to vascular deregulation leading to an impaired blood flow to the ONH. Later on, a new hypothesis involving the CSFP factor was appropriately formulated. A relatively low CSFP may generate mechanical conditions acting on the OHN similar to a relatively elevated IOP.

The first studies aimed to strengthen the hypothesis of glaucoma as a two-pressure disease were performed in cat and dog models several decades ago [72,73], but a large amount of clinical retrospective studies were later reported. Patients with high-pressure POAG were found to have lower CSF compared to normal patients [35,74]. Similarly, ocular hypertension patients showed higher CSFP compared to controls $[35,74]$. Interestingly, a positive correlation between translaminar pressure and patient's visual field loss was described [74]. In a more recent study, no significant differences between NTG patients and healthy controls with respect to CSFP or TLPG were reported [75]. However suggestive, all these studies included a limited number of subjects, which precluded strong statistical significance.

Noteworthy, several limitations should be considered in studying TLPG: i) while IOP is measured at the cornea, CSFP was generally measured at the lumbar level and not in the orbital space; ii) LC biomechanical properties largely depend on individuals differences that determine how susceptible the axons are to this stress; iii) changes in body position affect both CSFP and IOP so that studies performed by measuring patient's CSFP in a sitting position and ICP in supine position compromise TLPG evaluation and comparison; and iv) IOP and CSFP follow a circadian rhythm, therefore simultaneous measurement of IOP and ICP should be performed in order to obtain a reliable TLPG evaluation.

Numerous risk factors have been associated with the development of glaucoma and notably, some of them could be related to CSFP. These factors may affect CSFP as a potential risk factor for glaucoma. Generally, a positive correlation has been detected between increased Body Mass Index (BMI) and glaucoma, particularly in women [76-78]. Prospective and retrospective studies have shown that BMI is positively associated with CSFP in different populations [77-79]. The underlying hypothesis correlates obesity with an increased intra-abdominal and venous pressure, with a consequent increase in intracranial pressure [80]. Conversely, some reports support that lower BMI may increase the risk for glaucoma, or even that increased BMI may be protective $[81,82]$. These studies described an association only for women, suggesting that hormonal factors might be involved in regulating specific molecular pathways in glaucoma [83].

Age is a further well-documented risk factor for glaucoma. Although some studies that examined CSFP and age failed to find a relationship $[84,85]$, a recent study provided some evidences relating CSFP to age: CSFP was stable from ages 20-49 years, but progressively declined in the 6th decade. Interestingly, the age where CSFP begins to decline coincides with the age of prevalence increase in POAG [79]. Age-related decrease in CSFP could induce a relatively significant increase in the TLPG, thus supporting the idea that reduced CSFP may be a risk factor for POAG and NTG.
Finally, the impact of genetics in glaucoma is worthy of mention. It is well known that NPG has a high heritability. Two independent groups described an association between a single nucleotide polymorphisms (SNP) of the gene region containing the cyclin-dependent kinase inhibitor 2B antisense noncoding RNA (CDKN2B-AS1) and some glaucoma features among POAG patients [86,87]. Importantly, the CDKN2B-AS1 gene region is known to influence TGF-beta activity and to be actively transcribed by the CPE cells that secrete CSF.

\section{Overlapping genetic pathways control common functions in both brain and eye, in health and disease conditions}

As largely discussed above, pressure gradient at LC is a resultant of the net pressure force on the optic disk, determined by the difference between the ICP and IOP, which in turn depend, respectively, on CSF at retrobulbar space and aqueous humor $(\mathrm{AH})$ at the eye chamber.

CSF and AH are both produced by layers of neuroepithelial cells sealed by tight junctions: CSF is secreted from the choroid plexus epithelium (CPE), while $\mathrm{AH}$ is produced by the ciliary body epithelium (CBE). CBE is formed by two adjacent layers: the non-pigmented (NPE) and the pigmented epithelium (PE), sharing almost identical transcriptomic profiles [88]. In addition to produce colourless fluids involved in homeostatic regulation of CNS parenchyma or ocular tissues, and regulate pressure dynamics, the CPE and CBE share other common features [89]. Interestingly, 10 out of 65 POAG candidate disease genes were found highly expressed in both healthy human CPE and NPE, thus suggesting overlapping molecular mechanisms that could induce pathological pressure alterations in the two epithelia [90].

Another aspect associating CP and the visual system concerns the sophisticated mechanisms regulating the production and the homeostasis of both CSF and AH. In the same transcriptomic study, common expression profiles in CPE and NPE were mainly related to genes encoding ion channels and ion transporters, such as genes belonging to $A T P 1 A, K C N, S C N, A Q P$ and $S L C$ families, all playing a key role in CSF production. Moreover, ion channels and ion transporters are known to be essential to maintain retinal homeostasis and prevent retinal degeneration [91]. Thus, the genetic knockout of these genes in experimental animal models is expected to cause severe alterations in both $\mathrm{CP}$ and visual system. In fact, the targeted disruption of Slc4a5 (Slc4a5-/ $-)$, encoding the electrogenic sodium bicarbonate cotransporter NBCe2, results in abnormal structure of $\mathrm{CP}$ epithelial cells in mouse [92]. As a consequence, CSF pressure and composition are altered and the volume of lateral ventricles is reduced. Slc4a5-/ - mice also develop a severe retinopathy that results in visual impairment. Interestingly, Slc4a5 deletion affects the epithelial tissue in the eye, inducing rod outer segment tip displacement from the apical RPE plasma membranes, and a dense packaging of RPE apical microvilli. Moreover, ON degeneration could be indirect consequence of an increased TLPG.

Remarkably, CP and CB biological functions are both highly depending on the physiological contribution of ciliated cells. CPE-lining brain ventricles deploy hundreds of motile cilia to guarantee CSF circulation. On the other hand, the anterior portion of the ciliary body is characterized by ciliary structures protruding from CBE. Many other cells in the eye produce primary cilia (e.g. the retinal pigment epithelium cells, photoreceptors, Müller glia cells) and indeed, ciliated cells notably contribute to the vertebrate visual system function. Because of their abundant presence in the human body, genetic mutations resulting in abnormal formation or function of centrioles and cilia give rise to a wide group of disorders generically known as ciliopathies, exhibiting a wide range of phenotypes, such as diabetes, eye movement abnormalities, liver disease, hydrocephalus, infertility, cognitive impairment/defects, retinal degeneration. Emerging evidences from literature unearth a growing number of genes causing ciliopathies and several syndromic rare diseases, whose mutations are associated with both neurosensorial and CSF disorders, displaying pleiotropic phenotypic manifestations including hydrocephalia. Here we provide some 
examples of ciliopathy-associated genes that may be related to CSF diseases, such as CEP164, IFT88, BBS1 and TMEM67.

CEP164 belongs to the CEP family of centrosomal proteins, and is required for assembly of primary cilia. Mutations in CEP164 cause nephronophthisis-related ciliopathies, with a gradient of genotype/phenotype correlations in which null mutations cause severe dysplastic phenotypes, whereas hypomorphic alleles cause milder degenerative phenotypes. Interestingly, Cep164 conditional knockout in mice shows that this gene is essential in multiciliated cells because it modulates the selective transport of membrane vesicles and their cargoes into the ciliary compartment [93]. The IFT88 protein, a member of the tetratrico peptide repeat (TPR) family, is also involved in cilium biogenesis [94], and has been shown to be relevant for ciliary beating and CSF flow in zebrafish. Besides, IFT88 has been also localized in the primary cilium, both in renal cells and photoreceptors, and mutations in the Tg737 gene cause abnormal outer segment development, retinal degeneration and polycystic kidney disease in mouse [95]. On the other hand, $B B S 1$ is one of the genes causative of Bardet-Biedl syndrome-1, a genetic rare ciliopathy associated with a set of primary and secondary symptoms, such as retinitis pigmentosa, kidney dysfunction and behavioral dysfunction [96]. BBS1 is highly expressed in retina and the CSF, and a mouse mutant model show brain megaventricules and severe alterations in the CP cells. TMEM67 regulates centriole migration during primary cilium biogenesis, and mutations altering the multiple isoforms produced by TMEM67 result in ciliopathies, including retinal degeneration, renal cystic disease and a complex range of anatomical and functional defects of CNS, including hydrocephalia [97].

On the basis of these evidences, it is tempting to speculate that genes relevant in primary ciliogenesis are also key for multiciliogenesis. Pursuing the contribution of ciliopathy genes in CSF disorders is currently an unexplored but intriguing hypothesis.

\section{Conclusions}

Like human civilisations developing along riverbanks and seashores, vertebrate CNS develops around the extraordinarily dynamic and complex CSF. The strict mechanisms regulating the fine balance between secretion, composition, pressure and turnover of the primary fluid within the central nervous system have been refined during evolution. Alterations of this balance are implicated in a wide range of neurological pathologies: hydrocephalus, idiopathic intracranial hypertension, brain trauma, brain tumours and stroke, among others. The relevance of CSF in maintaining the healthy state of the eye, formed by a developmental evagination of the CNS and with a highly specialized function, is rapidly growing as extensively discussed in this review. However, researchers are still in the early stages of exploring how this information can be translated to relevant knowledge for new therapeutic treatments, particularly in glaucoma. Our current inability to measure cerebrospinal fluid pressure by non-invasive procedures and quantify the retrolaminar pressure gradient hinder advances in CSFPrelated eye diseases. Moreover, the precise cellular, molecular and physiological mechanisms that contribute to altered hydrodynamic pathways in ocular disorders are poorly understood. Notably, data originated from genomic and transcriptomic studies shed light on novel genes that could be related with the regulation of fluid flow in both CNS and neurosensorial structures. Often these are genes expressed by specialized cells located in different body compartments but sharing common functions. Consistently, inherited alterations of most of these genes that regulate nutrients and ion exchange, or liquid flow by adequate ciliary function alter a wide range of physiological processes and could lead to syndromic pathologies involving not only the brain and the neurosensorial systems, but also a number of organs such as kidney, heart or the reproductive system. Comprehensive studies on the biological processes compromised in patients suffering the pathological consequence of these molecular and cellular alterations will pave the way to design effective novel therapies for a wide range of CSF- associated disorders

\section{Funding}

This study was supported by grants BFU2017-86152-P to JFG, and SAF2016-80937-R (Ministerio de Economía y Competitividad/FEDER), 2017 SGR 738 (Generalitat de Catalunya), and La Marató TV3 (Project Marató 201417-30-31-32) to GM. SM was supported by a post-doctoral contract (ISCIII-CIBERER).

\section{Acknowledgments}

The authors would like to thank Izarbe Aísa-Marín for help in the preparation of figures.

\section{References}

[1] J.J. Iliff, M. Wang, D.M. Zeppenfeld, A. Venkataraman, B.A. Plog, Y. Liao, R. Deane, M. Nedergaard, Cerebral arterial pulsation drives paravascular CSF-interstitial fluid exchange in the murine brain, J. Neurosci. 33 (2013) 18190-18199, https://doi. org/10.1523/JNEUROSCI.1592-13.2013.

[2] R. Spector, R.F. Keep, S. Robert Snodgrass, Q.R. Smith, C.E. Johanson, A balanced view of choroid plexus structure and function: focus on adult humans, Exp. Neurol. 267 (2015) 78-86, https://doi.org/10.1016/j.expneurol.2015.02.032.

[3] L.F. Agnati, M. Marcoli, G. Leo, G. Maura, D. Guidolin, Homeostasis and the concept of "interstitial fluids hierarchy": relevance of cerebrospinal fluid sodium concentrations and brain temperature control (Review), Int. J. Mol. Med. 39 (2017) 487-497, https://doi.org/10.3892/ijmm.2017.2874.

[4] H. Davson, W.H. Oldendorf, Symposium on membrane transport. Transport in the central nervous system, Proc. R. Soc. Med. 60 (1967) 326-329 (Aaccessed August 21, 2019), http://www.ncbi.nlm.nih.gov/pubmed/6021942.

[5] K. Bräutigam, A. Vakis, C. Tsitsipanis, Pathogenesis of idiopathic normal pressure hydrocephalus: a review of knowledge, J. Clin. Neurosci. 61 (2019) 10-13, https:// doi.org/10.1016/j.jocn.2018.10.147.

[6] S.A. Liddelow, Fluids and barriers of the CNS: a historical viewpoint, Fluids Barriers CNS 8 (2011) 2, https://doi.org/10.1186/2045-8118-8-2.

[7] D. Bueno, J. Garcia-Fernàndez, Evolutionary development of embryonic cerebrospinal fluid composition and regulation: an open research field with implications for brain development and function, Fluids Barriers CNS 13 (2016) 5, https://doi. org/10.1186/s12987-016-0029-y.

[8] D. Orešković, M. Klarica, The formation of cerebrospinal fluid: nearly a hundred years of interpretations and misinterpretations, Brain Res. Rev. 64 (2010) 241-262, https://doi.org/10.1016/j. brainresrev.2010.04.006.

[9] Z.B. Redzic, J.E. Preston, J.A. Duncan, A. Chodobski, J. Szmydynger-Chodobska, The choroid plexus-cerebrospinal fluid system: from development to aging, Curr. Top. Dev. Biol. (2005) 1-52, https://doi.org/10.1016/S0070-2153(05)71001-2.

[10] R.F. Keep, H.C. Jones, A morphometric study on the development of the lateral ventricle choroid plexus, choroid plexus capillaries and ventricular ependyma in the rat, Dev. Brain Res. 56 (1990) 47-53, https://doi.org/10.1016/0165-3806(90) 90163-S.

[11] K.F. Chau, M.W. Springel, K.G. Broadbelt, H. Park, S. Topal, M.P. Lun, H. Mullan, T. Maynard, H. Steen, A.S. LaMantia, M.K. Lehtinen, Progressive differentiation and instructive capacities of amniotic fluid and cerebrospinal fluid proteomes following neural tube closure, Dev. Cell 35 (2015) 789-802, https://doi.org/10.1016/j. devcel.2015.11.015.

[12] G. Brocklehurst, The significance of the evolution of the cerebrospinal fluid system, Ann. R. Coll. Surg. Engl. 61 (1979) 349-356 (Accessed August 21, 2019), http:// www.ncbi.nlm.nih.gov/pubmed/386891.

[13] D. Bueno, J. Garcia-Fernàndez, Evolutionary development of embryonic cerebrospinal fluid composition and regulation: an open research field with implications for brain development and function, Fluids Barriers CNS 13 (2016) 5, https://doi. org/10.1186/s12987-016-0029-y.

[14] A.M. Smith, C.N. Czyz, Neuroanatomy, Cranial Nerve 2 (Optic) (2019) (Accessed October 9, 2019), http://www.ncbi.nlm.nih.gov/pubmed/29939684.

[15] R. Ren, J.B. Jonas, G. Tian, Y. Zhen, K. Ma, S. Li, H. Wang, B. Li, X. Zhang, N. Wang, Cerebrospinal fluid pressure in Glaucoma, Ophthalmology 117 (2010) 259-266, https://doi.org/10.1016/j.ophtha.2009.06.058.

[16] V. Promelle, J. Daouk, R. Bouzerar, B. Jany, S. Milazzo, O. Balédent, Ocular blood flow and cerebrospinal fluid pressure in glaucoma, Acta Radiol. Open 5 (2016) 205846011562427, , https://doi.org/10.1177/2058460115624275.

[17] Neurulation: coming to closure. - PubMed - NCBI, (n.d.). https://www.ncbi.nlm. nih.gov/pubmed/?term $=$ Neurulation $\% 3 \mathrm{~A}+$ coming + to + closure $($ Accessed October 9, 2019).

[18] A. Gato, M.E. Desmond, Why the embryo still matters: CSF and the neuroepithelium as interdependent regulators of embryonic brain growth, morphogenesis and histiogenesis, Dev. Biol. 327 (2009) 263-272, https://doi.org/10.1016/j.ydbio.2008. 12.029.

[19] M.W. Zappaterra, M.K. Lehtinen, The cerebrospinal fluid: regulator of neurogenesis, behavior, and beyond, Cell. Mol. Life Sci. 69 (2012) 2863-2878, https://doi.org/ 10.1007/s00018-012-0957-x. 
[20] M.D. Zappaterra, S.N. Lisgo, S. Lindsay, S.P. Gygi, C.A. Walsh, B.A. Ballif, A com parative proteomic analysis of human and rat embryonic cerebrospinal fluid, J. Proteome Res. 6 (2007) 3537-3548, https://doi.org/10.1021/pr070247w.

[21] L. Sakka, G. Coll, J. Chazal, Anatomy and physiology of cerebrospinal fluid, Eur. Ann. Otorhinolaryngol. Head Neck Dis. 128 (2011) 309-316, https://doi.org/10. 1016/j.anorl.2011.03.002.

[22] C. Nilsson, M. Lindvall-Axelsson, C. Owman, Neuroendocrine regulatory mechanisms in the choroid plexus-cerebrospinal fluid system., Brain Res. Brain Res. Rev. 17 (n.d.) 109-38. http://www.ncbi.nlm.nih.gov/pubmed/1393190 (Accessed August 21, 2019).

[23] M.G. Harrington, R.M. Salomon, J.M. Pogoda, E. Oborina, N. Okey, B. Johnson, D. Schmidt, A.N. Fonteh, N.F. Dalleska, Cerebrospinal fluid sodium rhythms, Cerebrospinal Fluid Res. 7 (2010) 3, https://doi.org/10.1186/1743-8454-7-3.

[24] K. Blennow, E. Vanmechelen, H. Hampel, CSF total tau, A 342 and phosphorylated tau protein as biomarkers for alzheimer's disease, Mol. Neurobiol. 24 (2001) 087-098, https://doi.org/10.1385/MN:24:1-3:087.

[25] J. Wiltfang, H. Esselmann, M. Bibl, A. Smirnov, M. Otto, S. Paul, B. Schmidt, H.W. Klafki, M. Maler, T. Dyrks, M. Bienert, M. Beyermann, E. Rüther, J. Kornhuber, Highly conserved and disease-specific patterns of carboxyterminally truncated A $\beta$ peptides 1-37/38/39 in addition to 1-40/42 in Alzheimer's disease and in patients with chronic neuroinflammation, J. Neurochem. 81 (2002) 481-496, https://doi. org/10.1046/j.1471-4159.2002.00818.x.

[26] A. Sakula, A hundred years of lumbar puncture: 1891-1991, J. R. Coll. Physicians Lond. 25 (1991) 171-175 (Accessed August 21, 2019), http://www.ncbi.nlm.nih. gov/pubmed/2066931.

[27] P.H. Raboel, J. Bartek, M. Andresen, B.M. Bellander, B. Romner, Intracranial pressure monitoring: invasive versus non-invasive Methods-A review, Crit. Care Res. Pract. 2012 (2012) 950393, , https://doi.org/10.1155/2012/950393.

[28] H.W. Querfurth, S.W. Arms, C.M. Lichy, W.T. Irwin, T. Steiner, Prediction of intracranial pressure from noninvasive transocular venous and arterial hemodynamic measurements: a pilot study, Neurocrit. Care 1 (2004) 183-194, https://doi.org/10. 1385/NCC:1:2:183.

[29] J.B. Jonas, N. Wang, S. Wang, Y.X. Wang, Q.S. You, D. Yang, W.B. Wei, L. Xu, Retinal vessel diameter and estimated cerebrospinal fluid pressure in arterial hypertension: the beijing eye study, Am. J. Hypertens. 27 (2014) 1170-1178, https:// doi.org/10.1093/ajh/hpu037.

[30] H. Yamasaki, M. Sugino, N. Ohsawa, Possible regulation of intracranial pressure by human atrial natriuretic peptide in cerebrospinal fluid, Eur. Neurol. 38 (1997) 88-93, https://doi.org/10.1159/000113166.

[31] C.F. Burgoyne, J.C. Downs, A.J. Bellezza, J.-K.F. Suh, R.T. Hart, The optic nerve head as a biomechanical structure: a new paradigm for understanding the role of IOP-related stress and strain in the pathophysiology of glaucomatous optic nerve head damage, Prog. Retin. Eye Res. 24 (2005) 39-73, https://doi.org/10.1016/j preteyeres.2004.06.001.

[32] C. Balaratnasingam, W.H. Morgan, V. Johnstone, S.S. Pandav, S.J. Cringle, D.-Y. Yu, Histomorphometric measurements in human and dog optic nerve and an estimation of optic nerve pressure gradients in human, Exp. Eye Res. 89 (2009) 618-628, https://doi.org/10.1016/j.exer.2009.06.002.

[33] J.B. Jonas, Role of cerebrospinal fluid pressure in the pathogenesis of glaucoma, Acta Ophthalmol. (Copenh) 89 (2011) 505-514, https://doi.org/10.1111/j.1755 3768.2010.01915.x.

[34] V.V. Volkov, [Essential element of the glaucomatous process neglected in clinical practice], Oftalmol. Zh. 31 (1976) 500-504 (Accessed August 21, 2019), http:// www.ncbi.nlm.nih.gov/pubmed/1012622.

[35] J.P. Berdahl, R.R. Allingham, D.H. Johnson, Cerebrospinal fluid pressure is decreased in primary open-angle glaucoma, Ophthalmology 115 (2008) 763-768, https://doi.org/10.1016/j.ophtha.2008.01.013.

[36] J.P. Berdahl, M.P. Fautsch, S.S. Stinnett, R.R. Allingham, Intracranial pressure in primary open angle Glaucoma, Normal tension Glaucoma, and ocular hypertension: a case-control study, Investig. Opthalmology Vis. Sci. 49 (2008) 5412, https://doi. org/10.1167/iovs.08-2228.

[37] L. Siaudvytyte, I. Januleviciene, A. Ragauskas, L. Bartusis, I. Meiliuniene, B. Siesky, A. Harris, The difference in translaminar pressure gradient and Neuroretinal Rim Area in Glaucoma and healthy subjects, J. Ophthalmol. 2014 (2014) 1-5, https:// doi.org/10.1155/2014/937360.

[38] T.H. Mader, C.R. Gibson, A.F. Pass, L.A. Kramer, A.G. Lee, J. Fogarty, W.J. Tarver, J.P. Dervay, D.R. Hamilton, A. Sargsyan, J.L. Phillips, D. Tran, W. Lipsky, J. Choi, C. Stern, R. Kuyumjian, J.D. Polk, Optic disc edema, globe flattening, choroidal folds, and hyperopic shifts observed in astronauts after long-duration space flight, Ophthalmology 118 (2011) 2058-2069, https://doi.org/10.1016/j.ophtha.2011. 06.021.

[39] L.A. Kramer, A.E. Sargsyan, K.M. Hasan, J.D. Polk, D.R. Hamilton, Orbital and intracranial effects of microgravity: findings at 3-T MR imaging, Radiology 263 (2012) 819-827, https://doi.org/10.1148/radiol.12111986.

[40] C.F. Burgoyne, A biomechanical paradigm for axonal insult within the optic nerve head in aging and glaucoma, Exp. Eye Res. 93 (2011) 120-132, https://doi.org/10. 1016/j.exer.2010.09.005

[41] H.A. Quigley, E.M. Addicks, Regional differences in the structure of the Lamina cribrosa and their relation to glaucomatous optic nerve damage, Arch. Ophthalmol. 99 (1981) 137-143, https://doi.org/10.1001/archopht.1981.03930010139020.

[42] J. Morrison, S. Farrell, E. Johnson, L. Deppmeier, C.G. Moore, E. Grossmann, Structure and composition of the rodent lamina cribrosa, Exp. Eye Res. 60 (1995) 127-135, https://doi.org/10.1016/s0014-4835(95)80002-6.

[43] M.R. Hernandez, F. Igoe, A.H. Neufeld, Cell culture of the human lamina cribrosa., invest, Ophthalmol. Vis. Sci. 29 (1988) 78-89 (Accessed August 21, 2019), http:// www.ncbi.nlm.nih.gov/pubmed/3275593.
[44] T. Tovar-Vidales, R.J. Wordinger, A.F. Clark, Identification and localization of lamina cribrosa cells in the human optic nerve head, Exp. Eye Res. 147 (2016) 94-97, https://doi.org/10.1016/j.exer.2016.05.006.

[45] I.C. Campbell, B. Coudrillier, C. Ross Ethier, Biomechanics of the posterior eye: a critical role in health and disease, J. Biomech. Eng. 136 (2014) 021005, , https:// doi.org/10.1115/1.4026286.

[46] J. Flammer, S. Orgül, V.P. Costa, N. Orzalesi, G.K. Krieglstein, L.M. Serra, J.P. Renard, E. Stefánsson, The impact of ocular blood flow in glaucoma, Prog. Retin. Eye Res. 21 (2002) 359-393 (Accessed August 21, 2019), http://www.ncbi.nlm. nih.gov/pubmed/12150988.

[47] C.F. Burgoyne, J. Crawford Downs, A.J. Bellezza, J.-K. Francis Suh, R.T. Hart, The optic nerve head as a biomechanical structure: a new paradigm for understanding the role of IOP-related stress and strain in the pathophysiology of glaucomatous optic nerve head damage, Prog. Retin. Eye Res. 24 (2005) 39-73, https://doi.org/ 10.1016/j.preteyeres.2004.06.001.

[48] W.-W. Su, S.-T. Cheng, W.-J. Ho, P.-K. Tsay, S.-C. Wu, S.H.L. Chang, Glaucoma is associated with peripheral vascular endothelial dysfunction, Ophthalmology 115 (2008) 1173-1178, https://doi.org/10.1016/j.ophtha.2007.10.026 e1.

[49] J.L. Son, I. Soto, E. Oglesby, T. Lopez-Roca, M.E. Pease, H.A. Quigley, N. MarshArmstrong, Glaucomatous optic nerve injury involves early astrocyte reactivity and late oligodendrocyte loss, Glia. 58 (2010), https://doi.org/10.1002/glia.20962 NA-NA.

[50] D.S. Minckler, M.O.M. Tso, L.E. Zimmerman, A. Light Microscopic Autoradiographic Study of Axoplasmic Transport in the Optic Nerve Head During Ocular Hypotony, Increased Intraocular Pressure, and Papilledema, Am. J. Ophthalmol. 82 (1976) 741-757, https://doi.org/10.1016/0002-9394(76) 90012-X.

[51] H. Quigley, D.R. Anderson, The dynamics and location of axonal transport blockade by acute intraocular pressure elevation in primate optic nerve, Invest. Ophthalmol. 15 (1976) 606-616 (Accessed August 21, 2019), http://www.ncbi.nlm.nih.gov/ pubmed/60300.

[52] W.H. Morgan, C.R.P. Lind, S. Kain, N. Fatehee, A. Bala, D.-Y. Yu, Author Response, Retinal vein pulsation is in phase with intracranial pressure and not intraocular pressure, Investig. Opthalmology Vis. Sci. 53 (2012) 6880, https://doi.org/10. 1167/iovs.12-10943.

[53] L. Dandona, A. Hendrickson, H.A. Quigley, Selective effects of experimental glaucoma on axonal transport by retinal ganglion cells to the dorsal lateral geniculate nucleus, Invest. Ophthalmol. Vis. Sci. 32 (1991) 1593-1599 (Accessed August 21, 2019), http://www.ncbi.nlm.nih.gov/pubmed/1707861.

[54] M. Salinas-Navarro, L. Alarcón-Martínez, F.J. Valiente-Soriano, M. Jiménez-López S. Mayor-Torroglosa, M. Avilés-Trigueros, M.P. Villegas-Pérez, M. Vidal-Sanz, Ocular hypertension impairs optic nerve axonal transport leading to progressive retinal ganglion cell degeneration, Exp. Eye Res. 90 (2010) 168-183, https://doi. org/10.1016/j.exer.2009.10.003.

[55] C. Balaratnasingam, W.H. Morgan, L. Bass, G. Matich, S.J. Cringle, D.-Y. Yu, Axona transport and cytoskeletal changes in the laminar regions after elevated intraocular pressure, Investig. Opthalmology Vis. Sci. 48 (2007) 3632, https://doi.org/10. 1167/iovs.06-1002.

[56] Y. Munemasa, Y. Kitaoka, J. Kuribayashi, S. Ueno, Modulation of mitochondria in the axon and soma of retinal ganglion cells in a rat glaucoma model, J. Neurochem. 115 (2010) 1508-1519, https://doi.org/10.1111/j.1471-4159.2010.07057.x.

[57] K.R.G. Martin, H.A. Quigley, D. Valenta, J. Kielczewski, M.E. Pease, Optic nerve dynein motor protein distribution changes with intraocular pressure elevation in rat model of glaucoma, Exp. Eye Res. 83 (2006) 255-262, https://doi.org/10.1016/ j.exer.2005.11.025.

[58] M.J. Barron, P. Griffiths, D.M. Turnbull, D. Bates, P. Nichols, The distributions of mitochondria and sodium channels reflect the specific energy requirements and conduction properties of the human optic nerve head, Br. J. Ophthalmol. 88 (2004) 286-290, https://doi.org/10.1136/bjo.2003.027664.

[59] W.-K. Ju, K.-Y. Kim, J.D. Lindsey, M. Angert, K.X. Duong-Polk, R.T. Scott, J.J. Kim, I. Kukhmazov, M.H. Ellisman, G.A. Perkins, R.N. Weinreb, Intraocular pressure elevation induces mitochondrial fission and triggers OPA1 release in glaucomatous optic nerve, Investig. Opthalmology Vis. Sci. 49 (2008) 4903, https://doi.org/10. 1167/iovs.07-1661.

[60] S. Baltan, D.M. Inman, C.A. Danilov, R.S. Morrison, D.J. Calkins, P.J. Horner, Metabolic vulnerability disposes retinal ganglion cell axons to dysfunction in a model of glaucomatous degeneration, J. Neurosci. 30 (2010) 5644-5652, https:// doi.org/10.1523/JNEUROSCI.5956-09.2010.

[61] Y. Dai, R.N. Weinreb, K.-Y. Kim, D. Nguyen, S. Park, X. Sun, J.D. Lindsey, M.H. Ellisman, W.-K. Ju, Inducible nitric oxide synthase-mediated alteration of mitochondrial OPA1 expression in ocular hypertensive rats, Investig. Opthalmology Vis. Sci. 52 (2011) 2468, https://doi.org/10.1167/iovs.10-5873.

[62] J.J. Iliff, M. Wang, Y. Liao, B.A. Plogg, W. Peng, G.A. Gundersen, H. Benveniste, G.E. Vates, R. Deane, S.A. Goldman, E.A. Nagelhus, M. Nedergaard, A paravascular pathway facilitates CSF flow through the brain parenchyma and the clearance of interstitial solutes, including amyloid, Sci. Transl. Med. 4 (2012), https://doi.org/ 10.1126/scitranslmed.3003748 147ra111-147ra111.

[63] J.J. Iliff, H. Lee, M. Yu, T. Feng, J. Logan, M. Nedergaard, H. Benveniste, Brain-wide pathway for waste clearance captured by contrast-enhanced MRI, J. Clin. Invest. 123 (2013) 1299-1309, https://doi.org/10.1172/JCI67677.

[64] J.J. Iliff, M.J. Chen, B.A. Plog, D.M. Zeppenfeld, M. Soltero, L. Yang, I. Singh, R. Deane, M. Nedergaard, Impairment of Glymphatic Pathway Function Promotes Tau Pathology after Traumatic Brain Injury, J. Neurosci. 34 (2014) 16180-16193, https://doi.org/10.1523/JNEUROSCI.3020-14.2014.

[65] N.A. Jessen, A.S.F. Munk, I. Lundgaard, M. Nedergaard, The glymphatic system: a beginner's guide, Neurochem. Res. 40 (2015) 2583-2599, https://doi.org/10.1007/ 
s11064-015-1581-6.

[66] A.K. Denniston, P.A. Keane, Paravascular pathways in the eye: is there an 'Ocular glymphatic system”?,' investig, Opthalmology Vis. Sci. 56 (2015) 3955, https://doi. org/10.1167/iovs.15-17243.

[67] T.N.C. Zeleny, C. Kohler, A. Neutzner, H.E. Killer, P. Meyer, Cell-Cell interaction proteins (Gap junctions, tight junctions, and desmosomes) and water transporter aquaporin 4 in meningothelial cells of the human optic nerve, Front. Neurol. 8 (2017) 308, https://doi.org/10.3389/fneur.2017.00308.

[68] I. Tsukahara, H. Yamashita, An electron microscopic study on the blood-optic nerve and fluid-optic nerve barrier., Albrecht Von Graefes Arch, Albrecht Von Graefes Arch. Klin. Exp. Ophthalmol. 196 (1975) 239-246 (Accessed August 22, 2019), http://www.ncbi.nlm.nih.gov/pubmed/1082256.

[69] E. Mathieu, N. Gupta, A. Ahari, X. Zhou, J. Hanna, Y.H. Yücel, Evidence for cerebrospinal fluid entry into the optic nerve via a glymphatic pathway, Investig. Opthalmology Vis. Sci. 58 (2017) 4784, https://doi.org/10.1167/iovs.17-22290.

[70] P. Wostyn, V. De Groot, D. Van Dam, K. Audenaert, H.E. Killer, P.P. De Deyn, The glymphatic hypothesis of Glaucoma: a unifying concept incorporating vascular, biomechanical, and biochemical aspects of the disease, Biomed Res. Int. 2017 (2017) 1-7, https://doi.org/10.1155/2017/5123148.

[71] L. Guo, T.E. Salt, V. Luong, N. Wood, W. Cheung, A. Maass, G. Ferrari, F. RussoMarie, A.M. Sillito, M.E. Cheetham, S.E. Moss, F.W. Fitzke, M.F. Cordeiro, Targeting amyloid-beta in glaucoma treatment, Proc. Natl. Acad. Sci. U. S. A. 104 (2007) 13444-13449, https://doi.org/10.1073/pnas.0703707104.

[72] M.E. Yablonski, An analysis of the \&quot;mechanical hypothesis\&quot; of glaucomatous optic disc cupping, Ann. Ophthalmol. 11 (1979) 427-428 (Accessed August 22, 2019), http://www.ncbi.nlm.nih.gov/pubmed/453746.

[73] J.E. Morgan, G. Jeffery, A.J.E. Foss, Axon deviation in the human lamina cribrosa, Br. J. Ophthalmol. 82 (1998) 680-683, https://doi.org/10.1136/bjo.82.6.680.

[74] R. Ren, J.B. Jonas, G. Tian, Y. Zhen, K. Ma, S. Li, H. Wang, B. Li, X. Zhang, N. Wang, Cerebrospinal fluid pressure in Glaucoma, Ophthalmology 117 (2010) 259-266, https://doi.org/10.1016/j.ophtha.2009.06.058.

[75] C. Lindén, S. Qvarlander, G. Jóhannesson, E. Johansson, F. Östlund, J. Malm, A. Eklund, Normal-tension Glaucoma Has normal intracranial pressure, Ophthalmology 125 (2018) 361-368, https://doi.org/10.1016/j.ophtha.2017.09. 022.

[76] P.A. Newman-Casey, N. Talwar, B. Nan, D.C. Musch, J.D. Stein, The relationship between components of metabolic syndrome and open-angle glaucoma, Ophthalmology 118 (2011) 1318-1326, https://doi.org/10.1016/j.ophtha.2010. 11.022 .

[77] R. Ren, N. Wang, X. Zhang, G. Tian, J.B. Jonas, Cerebrospinal fluid pressure correlated with body mass index, Graefes Arch. Clin. Exp. Ophthalmol. 250 (2012) 445-446, https://doi.org/10.1007/s00417-011-1746-1.

[78] J.P. Berdahl, D. Fleischman, J. Zaydlarova, S. Stinnett, R.R. Allingham, M.P. Fautsch, Body mass index has a linear relationship with cerebrospinal fluid pressure, Investig. Opthalmology Vis. Sci. 53 (2012) 1422, https://doi.org/10. 1167/iovs.11-8220.

[79] D. Fleischman, J.P. Berdahl, J. Zaydlarova, S. Stinnett, M.P. Fautsch, R.R. Allingham, Cerebrospinal fluid pressure decreases with older age, PLoS One 7 (2012) e52664, , https://doi.org/10.1371/journal.pone.0052664.

[80] H.J. Sugerman, E.J. DeMaria, W.L. Felton, M. Nakatsuka, A. Sismanis, Increased intra-abdominal pressure and cardiac filling pressures in obesity-associated pseudotumor cerebri, Neurology 49 (1997) 507-511, https://doi.org/10.1212/wnl.49. 2.507 .

[81] L.R. Pasquale, W.C. Willett, B.A. Rosner, J.H. Kang, Anthropometric measures and their relation to incident primary open-angle Glaucoma, Ophthalmology 117 (2010) 1521-1529, https://doi.org/10.1016/j.ophtha.2009.12.017.

[82] W.D. Ramdas, R.C.W. Wolfs, A. Hofman, P.T.V.M. de Jong, J.R. Vingerling, N.M. Jansonius, Lifestyle and risk of developing open-angle Glaucoma, Arch. Ophthalmol. 129 (2011) 767, https://doi.org/10.1001/archophthalmol.2010.373.

[83] O.A. Agapova, P.L. Kaufman, M.R. Hernandez, Androgen receptor and NFkB expression in human normal and glaucomatous optic nerve head astrocytes in vitro and in experimental glaucoma, Exp. Eye Res. 82 (2006) 1053-1059, https://doi. org/10.1016/j.exer.2005.10.021.

[84] M. Czosnyka, Z.H. Czosnyka, P.C. Whitfield, T. Donovan, J.D. Pickard, Age dependence of cerebrospinal pressure-volume compensation in patients with hydrocephalus, J. Neurosurg. 94 (2001) 482-486, https://doi.org/10.3171/jns.2001.
94.3.0482.

[85] J. Malm, J. Jacobsson, R. Birgander, A. Eklund, Reference values for CSF outflow resistance and intracranial pressure in healthy elderly, Neurology. 76 (2011) 903-909, https://doi.org/10.1212/WNL.0b013e31820f2dd0.

[86] L.R. Pasquale, S.J. Loomis, J.H. Kang, B.L. Yaspan, W. Abdrabou, D.L. Budenz, T.C. Chen, E. Delbono, D.S. Friedman, D. Gaasterland, T. Gaasterland, C.L. Grosskreutz, R.K. Lee, P.R. Lichter, Y. Liu, C.A. McCarty, S.E. Moroi, L.M. Olson, T. Realini, D.J. Rhee, J.S. Schuman, K. Singh, D. Vollrath, G. Wollstein, D.J. Zack, R.R. Allingham, M.A. Pericak-Vance, R.N. Weinreb, K. Zhang, M.A. Hauser, J.E. Richards, J.L. Haines, J.L. Wiggs, CDKN2B-AS1 genotype-glaucoma feature correlations in primary open-angle glaucoma patients from the United States, Am. J. Ophthalmol. 155 (2013) 342-353, https://doi.org/10.1016/j.ajo. 2012.07.023 e5.

[87] J.L. Wiggs, B.L. Yaspan, M.A. Hauser, J.H. Kang, R.R. Allingham, L.M. Olson, W. Abdrabou, B.J. Fan, D.Y. Wang, W. Brodeur, D.L. Budenz, J. Caprioli, A. Crenshaw, K. Crooks, E. Delbono, K.F. Doheny, D.S. Friedman, D. Gaasterland, T. Gaasterland, C. Laurie, R.K. Lee, P.R. Lichter, S. Loomis, Y. Liu, F.A. Medeiros, C. McCarty, D. Mirel, S.E. Moroi, D.C. Musch, A. Realini, F.W. Rozsa, J.S. Schuman, K. Scott, K. Singh, J.D. Stein, E.H. Trager, P. Vanveldhuisen, D. Vollrath, G. Wollstein, S. Yoneyama, K. Zhang, R.N. Weinreb, J. Ernst, M. Kellis, T. Masuda D. Zack, J.E. Richards, M. Pericak-Vance, L.R. Pasquale, J.L. Haines, Common variants at 9p21 and $8 \mathrm{q} 22$ are associated with increased susceptibility to optic nerve degeneration in glaucoma, PLoS Genet. 8 (2012) e1002654, , https://doi.org/ 10.1371/journal.pgen.1002654.

[88] S.F. Janssen, T.G.M.F. Gorgels, K. Bossers, J.B. ten Brink, A.H.W. Essing, M. Nagtegaal, P.J. van der Spek, N.M. Jansonius, A.A.B. Bergen, Gene expression and functional annotation of the human ciliary body epithelia, PLoS One 7 (2012) e44973, , https://doi.org/10.1371/journal.pone.0044973.

[89] M. Cocaprados, J. Escribano, New perspectives in aqueous humor secretion and in glaucoma: the ciliary body as a multifunctional neuroendocrine gland, Prog. Retin. Eye Res. 26 (2007) 239-262, https://doi.org/10.1016/j.preteyeres.2007.01.002.

[90] S.F. Janssen, T.G. Gorgels, J.B. Ten Brink, N.M. Jansonius, A.A. Bergen, Gene expression-based comparison of the human secretory neuroepithelia of the brain choroid plexus and the ocular ciliary body: potential implications for glaucoma, Fluids Barriers CNS 11 (2014) 2, https://doi.org/10.1186/2045-8118-11-2.

[91] D. Križaj, What Is Glaucoma? (1995) (Accessed August 22, 2019), http://www.ncbi. nlm.nih.gov/pubmed/31241881.

[92] L. Kao, L.M. Kurtz, X. Shao, M.C. Papadopoulos, L. Liu, D. Bok, S. Nusinowitz B. Chen, S.L. Stella, M. Andre, J. Weinreb, S.S. Luong, N. Piri, J.M.K. Kwong, D. Newman, I. Kurtz, Severe neurologic impairment in mice with targeted disruption of the electrogenic sodium bicarbonate cotransporter NBCe2 (Slc4a5 gene), J. Biol. Chem. 286 (2011) 32563-32574, https://doi.org/10.1074/jbc.M111.249961.

[93] S.S. Siller, H. Sharma, S. Li, J. Yang, Y. Zhang, M.J. Holtzman, W. Winuthayanon, H. Colognato, B.C. Holdener, F.-Q. Li, K.-I. Takemaru, Conditional knockout mice for the distal appendage protein CEP164 reveal its essential roles in airway multiciliated cell differentiation, PLoS Genet. 13 (2017) e1007128, , https://doi.org/10. 1371/journal.pgen.1007128.

[94] E.W. Olstad, C. Ringers, J.N. Hansen, A. Wens, C. Brandt, D. Wachten, E. Yaksi, N. Jurisch-Yaksi, Ciliary beating compartmentalizes cerebrospinal fluid flow in the brain and regulates ventricular development, Curr. Biol. 29 (2019) 229-241, https://doi.org/10.1016/j.cub.2018.11.059 e6.

[95] G.J. Pazour, S.A. Baker, J.A. Deane, D.G. Cole, B.L. Dickert, J.L. Rosenbaum, G.B. Witman, J.C. Besharse, The intraflagellar transport protein, IFT88, is essential for vertebrate photoreceptor assembly and maintenance, J. Cell Biol. 157 (2002) 103-113, https://doi.org/10.1083/jcb.200107108.

[96] R.E. Davis, R.E. Swiderski, K. Rahmouni, D.Y. Nishimura, R.F. Mullins, K. Agassandian, A.R. Philp, C.C. Searby, M.P. Andrews, S. Thompson, C.J. Berry, D.R. Thedens, B. Yang, R.M. Weiss, M.D. Cassell, E.M. Stone, V.C. Sheffield, A knockin mouse model of the Bardet Biedl syndrome 1 M390R mutation has cilia defects, ventriculomegaly, retinopathy, and obesity, Proc. Natl. Acad. Sci. 104 (2007) 19422-19427, https://doi.org/10.1073/pnas.0708571104.

[97] J.W. Shim, P.R. Territo, S. Simpson, J.C. Watson, L. Jiang, A.A. Riley, B. McCarthy, S. Persohn, D. Fulkerson, B.L. Blazer-Yost, Hydrocephalus in a rat model of Meckel Gruber syndrome with a TMEM67 mutation, Sci. Rep. 9 (2019) 1069, https://doi org/10.1038/s41598-018-37620-5. 